\title{
Effects of Exogenous Methyl Jasmonate Treatment with Polyethylene Bag Storage on Decay Reduction and Enhanced Total Ascorbic Acid, Total Phenolic, and Antioxidant Activities in 'Kamphaeng Saen 42' Mulberry Fruit
}

\author{
Laddawan Kammapana*, Siriporn Mulalin and Supawadee Tangteerawattana \\ Division of Plant Science Textiles and Design, Faculty of Agriculture and Technology, \\ Rajamangala University of Technology Isan, Surin campus, Surin 32000, Thailand
}

('Corresponding author's e-mail: noihort2526@gmail.com, Laddawan.ka@rmuti.ac.th)

Received: 20 October 2020, Revised: 28 May 2021, Accepted: 20 June 2021

\begin{abstract}
Mulberry fruit has a short shelf life of only 1 - 2 days under room conditions, make it one of the most fragile and extremely perishable fruits. This study investigated the effects of exogenous methyl jasmonate (MeJA) combined with polyethylene bag (PE) on postharvest decay, total ascorbic acid, total phenolic, anthocyanin, and antioxidant activities in 'Kamphaeng Saen 42' mulberry fruits. The study was conducted at Postharvest Quality and Storage System Laboratory, Division of AgriScience and Technology (Postharvest Technology), Faculty of Bioresources and Technology, King Mongkut's University of Technology Thonburi in December 2019. The experiment was laid out in Completely Randomized Design (CRD) with 4 replicates and 2 treatments consisting of 1) untreated with MeJA (control) and 2) fruits treated with $10 \mu \mathrm{mol} \mathrm{L}^{-1}$ methyl jasmonate (MeJA) combination with polyethylene bag $(\mathrm{PE})$ and maintained at $10 \pm 2{ }^{\circ} \mathrm{C}, 90 \pm 2 \%$ relative humidity $(\mathrm{RH})$. The results showed that treating the mulberry fruits with $10 \mu \mathrm{mol} \mathrm{L} \mathrm{L}^{-1}$ MeJA before placing them in the PE bag was the most effective method to reduce fruit decay and also resulted in higher levels of total ascorbic acid, total phenols, and antioxidant activities than in the control at the end of storage $(55.14 \mathrm{mg} / 100 \mathrm{~g} \mathrm{FW}, 43.78 \mathrm{mg} \mathrm{GAE} / \mathrm{g} \mathrm{FW}$ and $28.91 \mathrm{mM} \mathrm{TE} / 100 \mathrm{~g} \mathrm{FW}$ ), respectively. However, the treated fruits showed lower anthocyanin content than the control during the first 8 days of storage. These results suggest that the combination of MeJA with PE bag is beneficial in reducing fruit decay and increasing total ascorbic acid, total phenols, and antioxidant activities in 'Kamphaeng Saen 42 ' mulberry fruits during storage at $10 \pm 2{ }^{\circ} \mathrm{C}, 90 \pm 2 \%$ RH for 10 days.
\end{abstract}

Keywords: 'Kamphaeng Saen 42' mulberry fruit, Methyl jasmonate, Polyethylene bag, Decay, Antioxidant

\section{Introduction}

Kamphaeng Saen 42' mulberry is one of the mulberries grown to be eaten as fresh fruit, similar to the mulberry 'Chiang Mai 60'. Nowadays, this fruit is in high demand in the market because of its richness in bioactive components including vitamins, anthocyanins, alkaloids, flavonoids, and polyphenols [1]. The antioxidant compounds present in mulberry fruit, including vitamin $\mathrm{C}$, phenolic compounds, and anthocyanins have potential effects in reducing the risk of cardiovascular disease and cancer [2]. However, mulberry fruit is highly susceptible to physiological deterioration and pathogen attack, which limits its shelf life to $1-2$ days at room temperature.

Methyl jasmonate (MeJA) is an endogenous regulator that plays a role in plant growth and development and plant defense against biotic and abiotic stresses [3]. Recently, MeJA has been reported to effectively suppress postharvest diseases in various fruits, for example, blueberries [4], strawberries [5], longkong [6], and grapes [7]. Moreover, postharvest MeJA treatment preserved higher bioactive compounds content and improved antioxidant capacity in cherry tomatoes [8], raspberries [9], blueberries [10], jujube [11], and pineapple [12]. In addition, modified atmosphere packaging (MAP) is a practical way to create a suitable gaseous atmosphere around a fresh produce packaged in plastic film to extend shelf life and maintain produce quality. As a result, the atmospheric composition inside the package with a low concentration of $\mathrm{O}_{2}(<21 \%)$ and a high concentration of $\mathrm{CO}_{2}(>0.03 \%)$ reduces the respiration 
rate of fruits, delays fruit ripening, and suppresses diseases in many fruits [13]. However, the concentration of $\mathrm{O}_{2}$ and $\mathrm{CO}_{2}$ in the package depends on the respiration rate of the fruit, storage temperature, and permeability properties of the film material [14]. It has been reported that MAP is one of the most successful techniques for fruit preservation. Moreover, this method is simple and without chemical residues which is safe for human health [15].

Therefore, this research investigated the effect of exogenous methyl jasmonate (MeJA) combination with polyethylene bag (PE) on postharvest decay, total ascorbic acid, total phenol, anthocyanin, and antioxidant activities in 'Kamphaeng Saen 42' mulberry fruits.

\section{Materials and methods}

\section{Reagents and chemicals}

The reagents and chemicals used in this study were all of analytical grade and were obtained from Sigma-Aldrich, methyl jasmonate (Sigma, 95 \%), 2,2-diphenyl-1-picrylhydrazyl (Sigma, 98 \%), 2,4dinitrophenol (Sigma, 98 \%), 1-ascorbic acid (Sigma, 98 \%), gallic acid (Fluka, 98 \%), Folin-Ciocalteu reagent (Sigma, 2N), metaphosphoric acid (Sigma, $36.5 \%$ ), thiourea (Sigma, $99 \%$ ), sulfuric acid (Sigma, $98 \%$ ), 6-hydroxy-2,5,7,8-tetramethylchroman-2-carboxylic acid (Sigma, $98 \%$ ), potassium chloride (Sigma, $98 \%$ ), and sodium acetate trihydrate (Sigma, $98 \%$ ).

\section{Plant material}

Kamphaeng saen 42 ' mulberry fruit was hand-harvested at the red skin stage (70\% ripe) from Rajamangala Sericulture center, Rajamangala University of Technology Isan, Surin campus, and transported to Postharvest Quality and Storage System Laboratory. The fruits were selected based on uniformity of color and absence of defects or intactness and then randomly divided into 2 lots. All lots were placed in an airtight $45 \mathrm{~L}$ plastic container and fumigated with $10 \mu \mathrm{mol} \mathrm{L} \mathrm{LeJA}^{-1} \mathrm{Mnd}$ the untreated samples served as a control for $6 \mathrm{~h}$ at $20^{\circ} \mathrm{C}$. The MeJA concentration at $10 \mu \mathrm{mol} \mathrm{L} \mathrm{L}^{-1}$ was calculated based on the volume of the container. Then all treatments were packed in polyethylene bags (PE)(size $5 \times 8^{\prime \prime}$, with a thickness of $0.042 \mathrm{~mm}$, at oxygen transmission rate; OTR of $2,104 \mathrm{cc} \mathrm{m}^{-2} \mathrm{day}^{-1}$, and water vapor transmission rate (WVTR) of $14.8 \mathrm{~g} \mathrm{~m}^{-2}$ day $^{-1}$ ) and then kept at $10 \pm 2{ }^{\circ} \mathrm{C}, 90 \pm 2 \%$ relative humidity (RH). There were 4 replicates with approximately $500 \mathrm{~g}$ of fruit per replicate. Fruit samples were taken randomly from each treatment at 2-day intervals to determine postharvest decay, total ascorbic acid, total phenols, anthocyanins, and antioxidant activities.

\section{Fruit decay}

Fruit decay was assessed visually using 100 fruits from each replication. If more than $50 \%$ of visible mold growth occurred on the fruit surface, this was considered decayed. The severity of fruit decay was expressed as the percentage of fruits with fungal symptoms.

\section{Total ascorbic acid content}

Total ascorbic acid content was determined according to the method of Roe et al. [16]. Five grams of the sample was extracted with $20 \mathrm{~mL}$ of $5 \%$ metaphosphoric acid using a homogenizer in an ice bath. The extract was filtered using Whatman \# 01 filter paper. Two tenths $\mathrm{mL}$ of a $0.02 \%$ indophenol solution was added to $0.4 \mathrm{~mL}$ of the sample extract and incubated for 2 - 3 min until a stable reddish-pink color was obtained. Then, $0.4 \mathrm{~mL}$ of $2 \%$ thiourea and $0.2 \mathrm{~mL}$ of $2 \%$ DNP solution were added and then incubated for $3 \mathrm{~h}$ at $37^{\circ} \mathrm{C}$ in a hot water bath. Then $1 \mathrm{~mL}$ of $85 \%$ sulfuric acid was added and incubated again for $30 \mathrm{~min}$ at room temperature. The absorbance was determined at $540 \mathrm{~nm}$ using a $1601 \mathrm{UV}-$ visible spectrophotometer (Shimadzu, Tokyo, Japan). A standard curve was prepared using standard ascorbic acid with concentrations of $20,40,60,80$ and $100 \mathrm{mgL}^{-1}$

\section{Total phenolic content}

Total phenol content was determined according to the method of Cheung et al. [17] with slight modifications by mixing $1 \mathrm{~mL}$ of supernatant with $5 \mathrm{~mL}$ of Folin-Ciocalteu's phenol reagent $(10 \% \mathrm{v} / \mathrm{v})$. After $3 \mathrm{~min}, 4 \mathrm{~mL}$ of $7.5 \% \mathrm{Na}_{2} \mathrm{CO}_{3}$ was added to the mixture. The reaction was kept at $30{ }^{\circ} \mathrm{C}$ for $1 \mathrm{~h}$, after which the absorbance was measured at $760 \mathrm{~nm}$ using a $1601 \mathrm{UV}$-visible spectrophotometer. The results were expressed as gallic acid equivalents (GAE) in mg GAE /100 g FW. A standard curve was prepared using the above procedure with a range of gallic acid at $20,40,60,80$ and $100 \mathrm{mgL}^{-1}$ 


\section{Anthocyanin content}

Anthocyanin content was measured according to the method of Horwitz [18]. $500 \mathrm{mg}$ of the samples were homogenized in $20 \mathrm{~mL}$ of distilled water. This was followed by sonication $\left(40{ }^{\circ} \mathrm{C}\right.$ for 15 $20 \mathrm{~min}$ ) and then incubating at room temperature until the temperature of the samples decreased to room temperature. After centrifugation at $8,000 \mathrm{rpm}$ for $5 \mathrm{~min}, 3 \mathrm{~mL}$ of the supernatant was mixed with $7 \mathrm{~mL}$ of $0.025 \mathrm{M}$ potassium chloride buffer at $\mathrm{pH} 1$ and $3 \mathrm{~mL}$ of the supernatant was mixed with $7 \mathrm{~mL}$ of $0.4 \mathrm{M}$ sodium acetate buffer at $\mathrm{pH}$ 4. The absorbance was determined at 510 and $700 \mathrm{~nm}$ using a $1601 \mathrm{UV}-$ visible spectrophotometer. Anthocyanin content was calculated using the following equations;

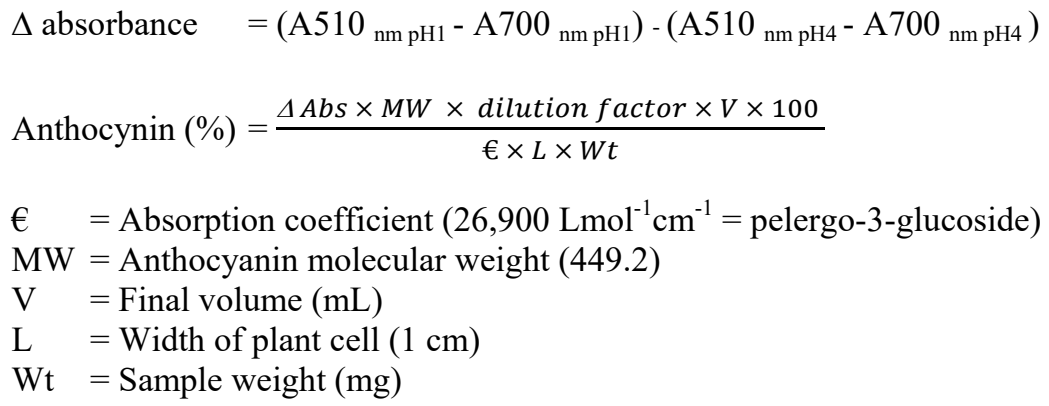

\section{Antioxidant activity (DPPH method)}

The DPPH assay was performed according to the method of Brand-Williams et al. [19] with slight modifications by reacting $150 \mu \mathrm{L}$ of fruit extracts with $2,850 \mu \mathrm{L}$ of the DPPH solution and then incubated at room temperature for $24 \mathrm{~h}$ in the dark. Then the absorbance was measured at $515 \mathrm{~nm}$. The standard curve was linear between 25 and $800 \mu \mathrm{M}$ Trolox.

\section{Statistical analysis}

The experimental design was a completely randomized design (CRD). All data analyses were performed using SAS statistical software (SAS Institute Inc. Cary, NC). Data were analyzed using a 1way analysis of variance (ANOVA). Mean separations were performed using Duncan's New Multiple Range Test (DMRT), and differences at $p \leq 0.05$ were considered statistically significant.

\section{Results and discussion}

\section{Fruit decay}

Mulberry fruit treated with $10 \mu \mathrm{mol} \mathrm{L} \mathrm{L}^{-1} \mathrm{MeJA}$ combination with PE bag were the most effective in controlling fruit decay compared to the control lot throughout the storage period. Meanwhile, the control lot showed fruit decay during the first 6 days of storage. At the end of storage, the decay incidence of MeJA combination with PE bag was only $8.60 \%$, while the control lot showed $14.83 \%$ fruit decay (Figure 1). It was reported that MeJA had effects against infection of fruit pathogens. It showed antifungal activity, inhibited spore production, spore germination and germ tube elongation, and induced plant defense against pathogens [20]. In addition, MAP was successful in retarding chilling injury (CI), color development, water loss, and fungal infection in various crops [21,22]. Gonzalez-Aguilar et al. [23] reported that papaya treated with $10^{-5} \mathrm{M}$ MeJA combination with MAP inhibited fruit decay and reduced CI development during storage for 14 - 32 days at $10{ }^{\circ} \mathrm{C}$ and 4 days shelf life at $20{ }^{\circ} \mathrm{C}$. These positive effects can be explained by the fact that MAP enhanced the efficacy of MeJA treatment. Therefore, the combination of MeJA with PE could delay fruit ripening and senescence, thereby reducing the decay of mulberry fruit in this study.

\section{Total ascorbic acid content}

Changes in total ascorbic acid content of mulberry fruit treated with $10 \mu \mathrm{mol}^{-1} \mathrm{MeJA}$ combination with PE bag and control are shown in Figure 2A. After 2 days of storage, there were no significant differences in total ascorbic acid content between treated fruits and the control. However, the content of total ascorbic acid in treated fruits at the end of storage was $55.14 \mathrm{mg} / 100 \mathrm{~g} \mathrm{FW}$ higher than that of the control $(29.81 \mathrm{mg} / 100 \mathrm{~g} \mathrm{FW})$. In general, total ascorbic acid or vitamin $\mathrm{C}$ is most sensitive to destruction when the commodity is exposed to unfavorable handling and storage conditions. Losses are exacerbated by prolonged storage, elevated temperatures, low relative humidity, physical damage, and exposure to cold. It has been reported that treatment with MAP and MeJA reduce CI, fungal attack, and water loss in 
various crops and also maintains or increases the production of secondary metabolites [24]. Similarly, the results of Ozturk et al. [25] reported that medlar fruits treated with $0.1 \mathrm{mM}$ MeJA combination with MAP reduced the loss of ascorbic acid due to a decrease in $\mathrm{O}_{2}$ concentration and increase in $\mathrm{CO}_{2}$ concentration in MAP which slow down the loss of ascorbic acid by inhibiting oxidation. Many studies have confirmed that MeJA also retards water loss, and induces antioxidant capacity of postharvest fruits [26]. In addition, a study on plant cell suspensions found that MeJA can induce the transcription of genes involved in the de novo biosynthesis of ascorbic acid [27]. Thus, the total ascorbic acid content of fruits treated with 10 $\mu \mathrm{mol}^{-1}$ MeJA combination with PE bag was higher than that of the control.

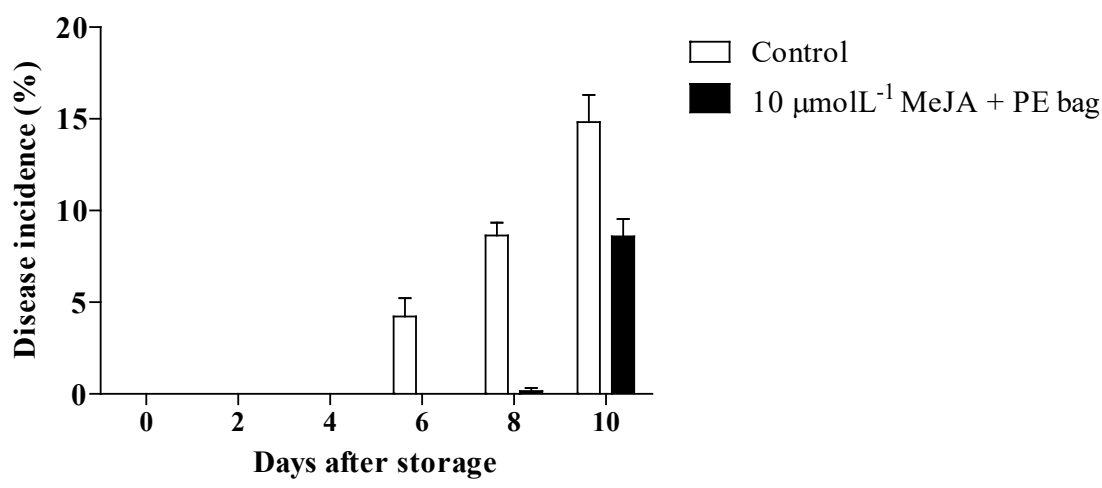

Figure 1 Disease incidence in 'Kamphaeng Saen 42' mulberry fruit treated with $10 \mu \mathrm{mol} \mathrm{L}{ }^{-1}$ MeJA combination with $\mathrm{PE}$ bag and control during storage at $10 \pm 2{ }^{\circ} \mathrm{C}, 90 \pm 2 \% \mathrm{RH}$ for 10 days.

\section{Total phenolic content}

Fruits treated with MeJA combination with PE bag had higher total phenolic content than the control throughout the storage period. At the end of storage, the total phenolic content of the treated fruits was $43.73 \mathrm{mg} / \mathrm{GAE} \mathrm{g} \mathrm{FW}$, while the total phenolic content in the control was $38.12 \mathrm{mg} / \mathrm{GAE} \mathrm{g}$ FW (Figure 2B). Wang et al. [10,28] found that MeJA treatment increased PAL activity in blueberries and Chinese bayberries, resulting in stimulation of phenol synthesis. It has been reported that MAP with low $\mathrm{O}_{2}$ and high $\mathrm{CO}_{2}$ delays the loss of total phenolic content by decreasing polyphenol oxidase activity in sweet cherry fruit [29] and loquat [21], as well as induces their abiotic stress, increasing phenolic compounds in the plant [30]. Thus, when MeJA was applied with MAP, it was the most effective in reducing the total phenolic content in mulberry fruit.

\section{Anthocyanin content}

Anthocyanin content was higher in the control than in the treated fruits during the first 8 days of storage, while the fruits treated with $10 \mu \mathrm{molL}^{-1} \mathrm{MeJA}$ combination with PE bag had the highest anthocyanin content $(34.42 \%$ ) at the end of storage (Figure 2C). Anthocyanins are colored water-soluble pigments found in mulberry fruit [31]. Many studies have shown that MeJA induces the accumulation of anthocyanins in many fruits [32,33]. However, our results showed that the combination of MeJA with PE bag had lower anthocyanin content than the control. This result suggests that the MAP technique delays fruit ripening and the effect of MeJA treatment on fruit ripening differs depending on the applied concentrations in different plants [32,34]. It was reported that fruits treated with MeJA at $5 \mu \mathrm{molL}^{-1}$ suppressed ethylene biosynthesis and delayed fruit ripening, while the promotion of fruit ripening with MeJA occurred in the range of $100 \mu \mathrm{molL}^{-1}$ to $1 \mathrm{mM}$ [35]. Feng et al. [36] reported that apples treated with 1 and $0.1 \mathrm{mM}$ MeJA promoted anthocyanin accumulation by up-regulating the expression of genes encoding enzymes involved in anthocyanin synthesis.

\section{Antioxidant activity}

Mulberry fruit treated with $10 \mu \mathrm{molL}^{-1}$ MeJA combination with PE bag exhibited significantly higher levels of antioxidant activity throughout the storage period (Figure 2D). At the storage, antioxidant activity of the treated fruits was $28.91 \mathrm{mM} \mathrm{TE} / 100 \mathrm{~g} \mathrm{FW}$, while the antioxidant activity in the control was $23.48 \mathrm{mM}$ TE $/ 100 \mathrm{~g} \mathrm{FW}$. In various berry fruits, MeJA can increase antioxidant activity through the increase of antioxidant compounds such as ascorbic acid, phenols and flavonoids [28,32,37,38]. Ozturk et al. [25] found that medlar treated with MeJA combination with MAP had the 
highest content of total phenols, and total flavonoids, which was also reflected in high antioxidant content. Similar results were found in this study.
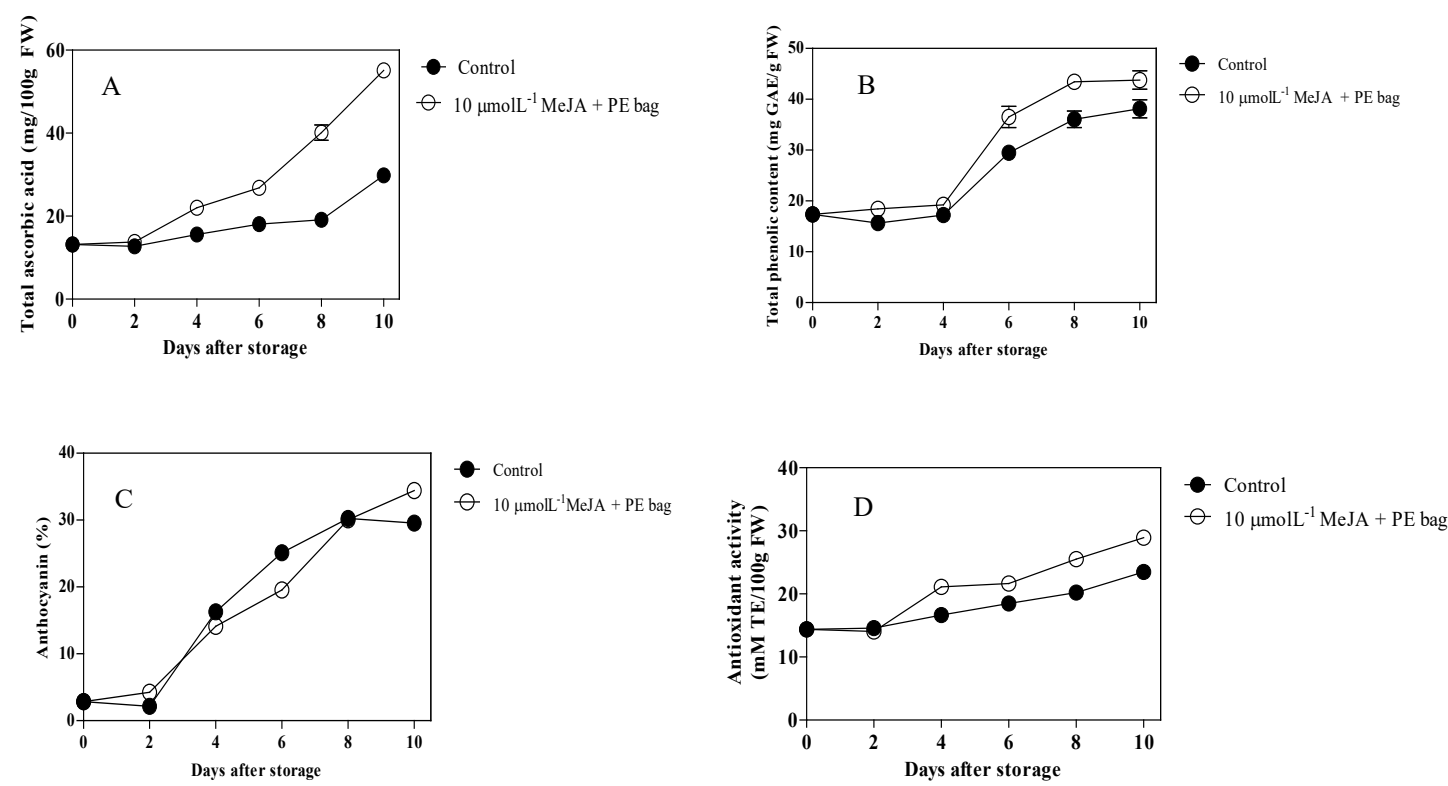

Figure 2 A) Changes in total ascorbic acid content, B) total phenolic content, C) anthocyanin content, and D) antioxidant activity of 'Kamphaeng Saen 42' mulberry fruit treated with $10 \mu \mathrm{mol} \mathrm{L}{ }^{-1} \mathrm{MeJA}$ combined with $\mathrm{PE}$ bag and control during storage at $10 \pm 2{ }^{\circ} \mathrm{C}, 90 \pm 2 \% \mathrm{RH}$ for 10 days.

\section{Conclusions}

Mulberry fruits treated with $10 \mu \mathrm{molL}^{-1} \mathrm{MeJA}$ combined with PE bag were the most effective in reducing fruit decay and resulted in higher levels of total ascorbic acid, total phenols and antioxidant activities than the control. Thus, the combination of MeJA with PE may improve the postharvest quality of 'Kamphaeng Saen 42' mulberry fruit during storage. Future studies on the activity of PAL and antioxidant enzyme activities, including SOD, CAT, and APX, should be conducted.

\section{Acknowledgements}

The authors would like to thank the Division of Plant Science Textiles and Design, Faculty of Agriculture and Technology, Rajamangala University of Technology Isan, Surin campus and Postharvest Quality and Storage System Laboratory, Division of AgriScience and Technology (Postharvest Technology), Faculty of Bioresources and Technology, King Mongkut's University of Technology Thonburi for providing the equipment required for samples testing. This research was supported by Rajamangala University of Technology Isan. Finally, we thank Professor Dr. Iftekhar Ahma from the Department of Food Engineering and Tea Technology. Shahjalal University of Science and Technology, Sylhet, Bangladesh for the help until the completion of this work.

\section{References}

[1] H Zhang, FW Zheng, L Xiaoqui and L Xinli. Effects of mulberry fruit (Morus alba L.) consumption on health outcomes: A mini-review. Antioxidants 2018; 7, 69.

[2] X Huang, J Li, H Shang and X Meng. Effect of methyl jasmonate on the anthocyanin content and antioxidant activity of blueberries during cold storage. J. Sci. Food Agr. 2015; 95, 337-43.

[3] L Bertini, S Proietti, F Focaracci, B Sabatin and C Carusco. Epigenetic control of dense gene following MeJA-induce priming in rice (O. sativa). J. Plant Physiol. 2018; 228, 166-17.

[4] H Wang, X Kou, C Wu, G Fan and T Li. Methyl jasmonate induces the resistance of postharvest blueberry to gray mold caused by Botrytis cinerea. J. Sci. Food Agr. 2020; 100, 4272-81.

[5] GM Saavedra, E Sanfuentes, PM Figueroa and CR Figueroa. Independent preharvest applications of methyl jasmonate and chitosan elicit differential upregulation of defense-related genes with reduced 
incidence of gray mold decay during postharvest storage of Fragaria chiloensis fruit. Int. J. Mol. Sci. 2017; 18, 1420.

[6] K Venkatachalam and M Mutita. Effect of methyl jasmonate on physiological and biochemical quality changes of longkong fruit under low temperature storage. Fruits 2015; 70, 69-75.

[7] L Jiang, P Jin, L wang, X Yu, H Wang and Y Zheng. Methyl jasmonate primes defense responses against Botrytis cinerea and reduces disease development in harvested table grapes. Sci. Hortic. 2015; 192, 218-23.

[8] H Liu, F Meng, H Miao, S Chen, T Yin, S Hu and Q Wang. Effects of postharvest methyl jasmonate treatment on main health-promoting components and volatile organic compounds in cherry tomato fruit. Food Chem. 2018; 263, 194-200.

[9] L Moro, NM Aymoto Hassimotto and E Purgatto. Postharvest auxin and methyl jasmonate effect on anthocyanin biosynthesis in red raspberry (Rubus idaeus L.). J. Plant Growth Regul. 2017; 36, 77382.

[10] H Wang, Y Wu, R Yu, C Wu, G Fan and T Li. Effects of postharvest application of methyl jasmonate on physicochemical characteristics and antioxidant system of the blueberry fruit. Sci. Hort. 2019; 258, 108785.

[11] Y Dong, HH Zhi, J Xu, LH Zhang, MP Liu and W Zong. Effect of methyl jasmonate on reactive oxygen species, antioxidant systems and microstructure of Chinese winter jujube at two major ripening stages during shelf life. J. Hort. Sci. Biotechnol. 2016; 91, 316-23.

[12] P Sangprayoon, S Supapvanich, P Youryon, C Wong-Aree and P Boonyaritthongchai. Efficiency of salicylic acid or methyl jasmonate immersions on internal browning alleviation and physicochemical quality of Queen pineapple cv. "Sawi" fruit during cold storage. J. Food Biochem. 2019; 43, 13059.

[13] Y Li, Y Ishikawa, T Satake, H Kitazawa, X Qiu and S Rungchang. Effect of active modified atmosphere packaging with different initial gas compositions on nutritional compounds of shiitake mushrooms (Lentinus edodes). Postharvest Biol. Tech. 2014; 92, 107-13.

[14] S Kartal, S Aday and C Caner. Use of microperforated films and oxygen scavengers to maintain storage stability of fresh strawberries. Postharvest Biol. Tech. 2012; 71, 32-40.

[15] OJ Caleb, PV Mahajan, FA Al-Said and UM Opera. Modified atmosphere packaging technology of fresh and fresh-cut produce and the microbial consequences: A review. Food Bioproc. Tech. 2013; 6, 303-29.

[16] JH Roe, BM Mary, MJ Oesterling and MD Charlotle. The determination of diketo L-gulonic acid, dehydro-L-ascorbic acid and L-ascorbic acid in the same tissue extract by 2,4-Dinitrophenyl hydrazine method. J. Biol. Chem. 1948; 174, 201-8.

[17] LM Cheung, PC Cheung and VE Ooi. Antioxidant activity and total phenolic of edible mushroom extract. J. Agr. Food Chem. 2003; 81; 249-55.

[18] W Horwitz. Official methods of analysis of AOAC international. $16^{\text {th }}$ ed. AOAC International, Gaithersburg MD, 2000, p. 1401.

[19] W Brand-Williams, ME Cuvelier and C Berset. Use of free radical method to evaluate antioxidant activity. Lebensm. Wiss. Tech. 1995; 28, 25-30.

[20] N Tzortzakis, A Chrysargyris, D Sivakumar and K Loulakakis. Vopour or dipping applications of methyl jasmonate, vinegar and sage oil for pepper fruit sanitation towards grey mold. Postharvest Biol. Tech. 2016; 118, 120-7.

[21] N Selcuk and M Erkan. The effects of modified and palliflex controlled atmosphere storage on postharvest quality and composition of 'Istanbul' medlar fruit. Postharvest Biol. Tech. 2015; 99, 919.

[22] R Porat, I Kosto and A Daus. Bulk storage of 'Wonderful' pomegranate fruit using modified atmosphere bags. Israel J. Plant Sci. 2016; 63, 45-50.

[23] GA Gonzalez-Aguilar, JG Buta and CY Wang. Methyl jasmonate and modified atmosphere packaging (MAP) reduce decay and maintain postharvest quality of papaya 'Sunrise'. Postharvest Biol. Tech. 2003; 28, 361-70

[24] H Yao and S Tian. Effects of pre-and post-harvest administration of salicylic acid of methyl jasmonate on inducing disease resistance of sweet cherry fruit in storage. Postharvest Biol. Tech. $2005 ; 35,253-62$.

[25] A Ozturk, K Yildiz, O Burhan and O Karakaya. Maintaining postharvest quality of medlar (Mespilus germanica) fruit using modified atmosphere packaging and methyl jasmonate. LWT Food Sci. Tech. 2019; 111, 117-24. 
[26] SY Wang, XC Shi, FQ Liu and P Laborda. Effects of exogenous methyl jasmonate on quality and preservation of postharvest fruits: A review. Food Chem. 2021; 353, 129482.

[27] BA Wolucka, A Goossens and D Inze. Methyl jasmonate stimulates the de no vo biosynthesis of vitamin C in plant cell suspensions. J. Exp. Bot. 2005; 56, 2527-38.

[28] K Wang, P Jin, S Cao, H Shang, Z Yang and Y Zheng. Methyl jasmonate reduces decay and enhances antioxidant capacity in Chinese bayberries. J. Agr. Food Chem. 2009; 57, 5089-815.

[29] O Ozkaya, A Sener, MA Saridas, U Unal, A Valizadeh and O Dundar. Influence of fast cold chain and modified atmosphere packaging storage on postharvest quality of early season-harvested sweet cherries. J. Food Proc. Preserv. 2015; 39, 2119-28.

[30] DO Kim, H Heo, Y kim, H Yang and CY Lee. Sweet and sour cherry phenolics and their protective effects on neuronal cell. J. Agr. Food Chem. 2005; 53, 9921-7.

[31] HE Kong, A Azlan, ST Tang and SM Lim. Anthocyanidins and anthocyanins: Colored pigments as food, pharmaceutical ingredients, and the potential health benefits. Food Nutr. Res. 2017; 61, 1361779.

[32] ME Garcia-Pastor, M Serrano, F Guillen, S Castillo, D Martinez-Romero and D Velero. Methyl jasmonate effects on table grape ripening, vine yield, berry quality and bioactive compound depend on applied concentration. Sci. Hortic. 2019; 247, 380-9.

[33] V Serna-Escolano, JM Valverde, ME Garcia-Pastor, D Valero, S Castillo and F Guillen. Pre-harvest methyl jasmonate treatments increase antioxidant systems in lemon fruit without affecting yield or other fruit quality parameters. J. Sci. Food Agr. 2019; 99, 5035-43.

[34] J Wei, X Wen and L Tang. Effect of methyl jasmonic acid on peach fruit ripening progress. Sci. Hortic. 2017; 220, 206-13.

[35] L Fan, J Shi, J Zho, L Gao, J Lv and Q Wang. Methyl jasmonate delays postharvest ripening and senescence in the non-climacteric eggplant (Solamun melongena L.) fruit. Postharvest Biol. Tech. 2016; 120, 76-83.

[36] S Feng, J Sun, S Sun, Y Wang, C Tian, Q Sun and X Chen. Transcriptional profiles underlying the effects of methyl jasmonate on apple ripening. J. Plant Growth Regul. 2017; 36, 271-80.

[37] PE Zuniga, Y Castaneda, O Arrey-Salas, L Fuentcs, F Aburto and CR Figueroa. Methyl jasmonate applications from flowering to ripe fruit stages of strawberry (Fragaria $x$ ananassa 'Camarosa') reinforce the fruit antioxidant response at post-harvest. Front. Plant Sci. 2020; 11, 538.

[38] G Flores and MLRD Castillo. Accumulation of anthocyanin and flavonols in black currants (Ribes nigrum L.) by pre-harvest methyl jasmonate treatments. J. Sci. Food Agr. 2016; 96, 4026-31. 